\title{
Viscoelastic properties of oxide-coated liquid metals
}

\section{Citation}

Larsen, Ryan J., Michael D. Dickey, George M. Whitesides, and David A. Weitz. 2009. “Viscoelastic Properties of Oxide-Coated Liquid Metals." Journal of Rheology53 (6): 1305-26. https:// doi.org/10.1122/1.3236517.

\section{Permanent link}

http://nrs.harvard.edu/urn-3:HUL.InstRepos:41511264

\section{Terms of Use}

This article was downloaded from Harvard University's DASH repository, and is made available under the terms and conditions applicable to Other Posted Material, as set forth at http:// nrs.harvard.edu/urn-3:HUL.InstRepos:dash.current.terms-of-use\#LAA

\section{Share Your Story}

The Harvard community has made this article openly available.

Please share how this access benefits you. Submit a story.

Accessibility 


\title{
Viscoelastic properties of oxide-coated liquid metals
}

\author{
Ryan J. Larsen ${ }^{\text {a) }}$ \\ School of Engineering and Applied Sciences, Harvard University, \\ 29 Oxford Street, Cambridge, Massachusetts 02138 \\ Michael D. Dickey ${ }^{\text {b) }}$ and George M. Whitesides \\ Department of Chemistry and Chemical Biology, Harvard University, \\ 12 Oxford Street, Cambridge, Massachusetts 02138 \\ David A. Weitz ${ }^{\text {) }}$ \\ School of Engineering and Applied Sciences, and Department of Physics, \\ Harvard University, 29 Oxford Street, Cambridge, Massachusetts 02138
}

(Received 14 October 2008; final revision received 20 August 2009)

\begin{abstract}
Synopsis
Many liquid metals exposed to air develop an oxide film on their outer surface. This film is sufficiently solid-like to provide mechanical stability to small liquid metal droplets, yet weak enough to allow the droplets to be malleable. These properties are useful in both micro-electronics and microfluidics; however, little is known about how to characterize them. Here we probe the elastic, yielding, and relaxation properties of oxide-coated gallium and eutectic gallium indium using a rheometer equipped with a parallel-plate geometry. By using parallel plates of different size, we show that surface stresses dominate bulk stresses. These experiments also demonstrate that the apparent elastic properties of the oxide film are highly sensitive to its strain history. Moreover, the apparent elasticity is sensitive to the stresses stored in the oxide skin. We probe these stresses and their time-dependence, with both torque and normal force measurements. We also characterize the time-dependence of the elasticity by observing free vibrations of the rheometer. We rationalize the strain history and time-dependence in terms of oxidation and show that despite this dependence, reproducible elasticity measurements can be obtained due to the ability of shear to produce a state that is independent of the strain history. (C) 2009 The Society of Rheology. [DOI: 10.1122/1.3236517]
\end{abstract}

\section{INTRODUCTION}

Small droplets of simple liquids tend to be spherical because this shape minimizes their surface area and surface free energy. Liquid droplets, and even bubbles, can deviate

\footnotetext{
${ }^{a)}$ Present address: Department of Chemical and Bimolecular Engineering, University of Illinois UrbanaChampaign, 114 Roger Adams Lab, 600 South Mathews, Urbana, Illinois 61801.

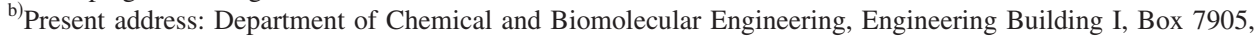
911 Partners Way, Raleigh, North Carolina 27695.

${ }^{c)}$ Author to whom correspondence should be addressed; electronic mail: weitz@seas.harvard.edu
} 
from this behavior when solids are incorporated onto their surface, thereby "solidifying" the liquid surface [Subramaniam et al. (2005); Xu et al. (2005)]. A similar realization of this principle occurs when the outer surface of a low-viscosity liquid metal is solidified, in atmosphere, by an oxide film. The film either slows or prevents further oxidation of the bulk liquid and allows metal droplets to be formed into shapes, such as cones, that would not ordinarily be favored due to surface tension [Chiechi et al. (2008); Dickey et al. (2008)]. These properties have been useful for making soft electrical contacts and for enhancing the stability of the liquid when it is injected into microfluidic devices [Chiechi et al. (2008); Dickey et al. (2008)].

To quantify the effect of oxidation on material properties, previous researchers have focused on surface tension measurements [Eustathopoulos and Drevet (1998); Ricci et al. (2005)]. Such measurements have limited usefulness for understanding the mechanical properties of the oxide skins in air, as demonstrated by recent experiments of flow of liquid metal in microchannels [Dickey et al. (2008)]. The liquid-like characteristics of eutectic gallium indium (EGaIn) were most apparent when the material was injected at constant pressure into a microfluidic channel that became progressively narrower. The narrowing of the channel caused the meniscus of the EGaIn to stop at a position that depended on the applied pressure. Advancing the meniscus further required higher applied pressure. A force balance between the pressure and the surface stress in the static meniscus revealed the maximum surface stress $\sigma_{D Y}$ that the oxide skin could support without flowing. For EGaIn, $\sigma_{D Y}=0.630 \mathrm{~N} / \mathrm{m}$, a value that is in excellent agreement with a separate measurement of an apparent surface tension, in atmosphere, obtained using the pendant drop method $(0.624 \mathrm{~N} / \mathrm{m})$ [Zrnic and Swatik (1969)]. This result implies that for this particular case, EGaIn can be characterized in terms of surface tension, a liquid-like characteristic. In this respect, EGaIn is not unlike mercury $(\mathrm{Hg})$, a low-viscosity liquid metal that does not oxidize in atmosphere: measurements of $\sigma_{D Y}$ obtained with $\mathrm{Hg}$ in the microfluidic device also agree with literature values of its surface tension (0.450 and $0.480 \mathrm{~N} / \mathrm{m}$, respectively) [Dickey et al. (2008)]. However, despite the similarity in how these two materials flow into microchannels, EGaIn and $\mathrm{Hg}$ exhibited qualitatively different behavior when the pressure was removed. The $\mathrm{Hg}$ rapidly retracted from the microfluidic channel to assume a shape that was less elongated and therefore more energetically favorable. By contrast, atmosphere-exposed EGaIn remained stationary in the channel. We attributed this stability to the oxide skin because when we removed the oxide skin by exposing the leading interface of EGaIn with hydrochloric acid, the EGaIn behaved like $\mathrm{Hg}$ and rapidly retracted from the channel. The mechanical stability of the oxide-coated EGaIn suggests that $\sigma_{D Y}$ should not be interpreted as the surface tension of a liquid but may instead be interpreted as a surface yield stress. When the dilational surface stress $\sigma_{D}$ is less than $\sigma_{D Y}$, the stress is stored elastically in the oxide skin. When $\sigma_{D} \geq \sigma_{D Y}$, the oxide skin will yield, or flow.

The yield stress character of the oxide skin was validated in experiments performed on a commercial rheometer, equipped with parallel plate geometries of different size [Dickey et al. (2008)]. The use of parallel plate geometries allows us to demonstrate that the stress required to deform small quantities of EGaIn originates from the oxide surface rather than the bulk and that the oxide film exhibits yield stress behavior. While performing these experiments, we also learned that, surprisingly, the elastic characteristics of the skin are highly dependent on its shear history: the apparent shear elasticity of the skin can be reversibly changed by an order of magnitude simply by changing the deformation history of the sample. This unusual effect has the potential to significantly complicate any future 
measurements of the mechanical properties of the oxide film. Here we present the results of a detailed study of these effects, as well as further investigations into the mechanisms responsible for the dependence on strain history.

These experiments elucidate some of the basic complications associated with performing mechanical measurements on oxidizing films. These complications include not only strain-history dependence but time-dependence as well. We show that for oxide surfaces that are not under stress, the apparent elasticity tends to increase with time, perhaps due to the effect of oxidation. However, when the oxide surface is allowed to relax while subjected to stress, the apparent elasticity tends to decrease in time and this effect is likely responsible for the observed dependence on strain history. We rationalize this result by proposing that the combination of stress, time, and oxidation causes the surface area of the oxide skin to increase. The "wrinkled" oxide skin is more easily deformed than the "taut" oxide skin, and elasticity measurements performed in the wrinkled state do not reflect true property values. Such effects have the potential of significantly complicating any rheological measurement of the properties of the oxide film. Nevertheless, our results suggest that shear deformation is able to "rejuvenate" the oxide skin and bring it to a reproducible and taut state, regardless of its shear and time history.

\section{EXPERIMENTAL}

Our experiments were conducted using three types of stress-controlled rheometers: the AR-G2 (TA Instruments), the C-VOR, and the Bohlin Gemini HRnano rheometer (both from Malvern Instruments). The rheometers were equipped with parallel-plate geometries of different size. In this configuration, the bottom plate is stationary and the upper plate is rigidly attached to a low-friction bearing. The upper plate is rotated by a motor that applies a torque $\tau$ to the bearing. When the sample is loaded between the two plates, the stress $\sigma$ in the sample is assumed to be proportional to $\tau$, and the strain $\gamma$ is assumed to be proportional to the bearing displacement $\theta$. In most of the experiments with the AR-G2, the upper plate material was made of hard anodized aluminum, and a homemade stainless steel plate covered the lower plate. In the case of the Malvern rheometers, both the upper and lower plates consisted of stainless steel. Our results did not appear to depend significantly on the choice of the materials used.

For our experiments, we used EGaIn and Ga (both $99.99 \%$ purity) as received from Sigma-Aldrich. Both materials are known to have skins consisting of oxides of Ga. As with previous experiments, the materials were used as received from the suppliers [Dickey et al. (2008)]. A syringe was used to place samples on the lower plate. The upper plate was then slowly lowered onto the sample while rotating slowly to aid with sample spreading. The gaps typically used varied from about 600 to $1300 \mu \mathrm{m}$ depending on the amount of sample loaded. For a given sample volume, we lowered the upper plate until the sample filled the gap between the plates. When the plates were lowered beyond this point, excessive "bulging" of the sample caused spherical-capped appendages to churn out from under the plates. Experiments were performed at $25^{\circ} \mathrm{C}$. Experiments on each sample were usually carried out within $24-48 \mathrm{~h}$ of loading.

\section{RESULTS}

\section{A. Surface elasticity}

Our first experiments are designed to test the hypothesis that the mechanical response of EGaIn is dominated by the yield stress character of the oxide skin. This is done by 
(a)

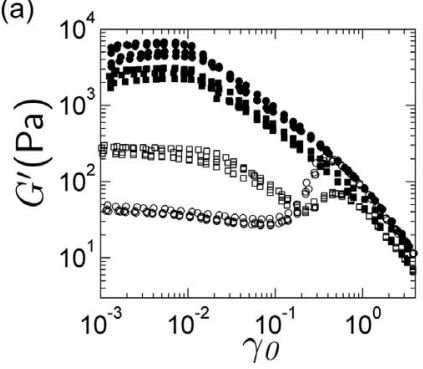

(b)

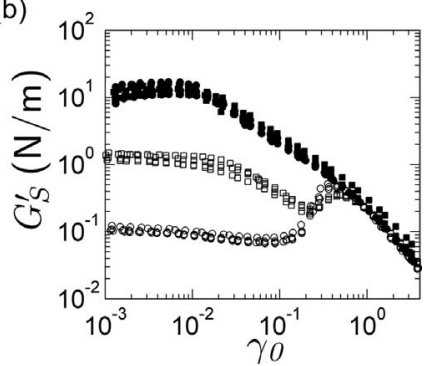

FIG. 1. Strain-controlled oscillatory measurements on EGaIn. Ascending (closed) and descending (open) strain amplitude sweeps performed with parallel plates of $20 \mathrm{~mm}$ (circles) and $40 \mathrm{~mm}$ (squares). (a) Measurements of the bulk elastic modulus $G^{\prime}$ are not consistent for the different plate sizes, but (b) measurements of the apparent surface elastic modulus $G_{S}^{\prime}$ are consistent for different plate sizes, as long as the material is in the stiff state (closed symbols), which is probed immediately following the pre-shear.

performing two sets of identical experiments using parallel plate geometries of different size. These experiments confirm that the oxide skin exhibits both the elastic and yield stress behaviors.

These measurements were performed on EGaIn using the HRnano equipped with parallel plates of 20 and $40 \mathrm{~mm}$ diameter. The rheometer performed strain oscillations at $\omega=1 \mathrm{rad} / \mathrm{s}$ of first ascending and then descending strain amplitude $\gamma_{0}$ and calculated apparent linear viscoelastic moduli $\left(G^{\prime}\right.$ and $\left.G^{\prime \prime}\right)$. We performed these experiments four times for each plate radius, with each test preceded by a strain-rate-controlled pre-shear $\left( \pm 1.3\right.$ or $\left.\pm 13 \mathrm{~s}^{-1}\right)$, applied for $5 \mathrm{~min}$ with no equilibration time before the oscillatory strain measurements. For both sets of tests, the gap was set at $1190 \mu \mathrm{m}$.

For $\gamma_{0}<0.01, G^{\prime} \gg G^{\prime \prime}$, and the moduli are roughly independent of $\gamma_{0}$. This corresponds to a linear, or near-linear, elastic regime [closed symbols, Fig. 1(a)]. As $\gamma_{0}$ is increased from 0.01 to $13, G^{\prime}$ decreases and the sample begins to exhibit dissipation or yielding. After ascending to a maximum value of $13, \gamma_{0}$ is subsequently decreased. For $\gamma_{0}=13$ to 0.5 , values of $G^{\prime}$ (open symbols in Fig. 1) are similar to those measured during the oscillations of ascending $\gamma_{0}$. Below $\gamma_{0}$ of $\sim 0.5$, however, the linear elasticity obtained from oscillations of descending $\gamma_{0}$ is significantly less than that measured during the oscillations of ascending $\gamma_{0}$. The material therefore appears to be stiffer following unidirectional pre-shear (stiff state) and less stiff following oscillations of decreasing $\gamma_{0}$ (soft state). Our results showed no systemic changes with time: the transition between the stiff and soft states is completely reversible. Moreover, values of $G^{\prime}$ exhibited no systemic dependence on the magnitude of the pre-shear.

In both the stiff and soft states, measured values of $G^{\prime}$ depend on the radius of the geometry as shown in Fig. 1(a). This suggests that the observed elastic response may originate from the surface stress component $\sigma_{S}$ that originates from the oxide skin and resists the torque by exerting a tangential force on the edge of the plate. The expected torque contribution $\tau_{S}$ due to $\sigma_{S}$ can be calculated by integrating $\sigma_{S}$ around the circumference $l$ of the plate of radius $R$,

$$
\tau_{S}=\int \sigma_{S} R d l=2 \pi \sigma_{S} R^{2}=\sigma_{S} / F_{S \sigma} .
$$

By contrast, the expected torque contribution $\tau_{B}$ from a bulk stress $\sigma$ is given by the standard conversion factor for a parallel plate rheometer, 


$$
\tau_{B}=\sigma \pi R^{3} / 2=\sigma / F_{B \sigma} .
$$

The ratio of surface to bulk torque $\tau_{S} / \tau_{B}$ defines the Boussinesq number Bo. When Bo $\ll 1$, surface stresses are negligible and rheometer-determined values of $G^{\prime}$ and $G^{\prime \prime}$ reflect the properties of the bulk material. However, it is unlikely that the bulk stresses of EGaIn are significant in our measurements because EGaIn is a Newtonian liquid of low viscosity ( $2 \mathrm{mPa}$ s for EGaIn) [Koster (1999)]. In the limit of $\mathrm{Bo} \gg 1$, it is possible to rescale the measured $G^{\prime}$ and $G^{\prime \prime}$ to obtain apparent surface viscoelastic shear moduli $G_{S}^{\prime}$ and $G_{S}^{\prime \prime}$. The scale of the surface moduli is set by $\sigma_{S} / \gamma$, where the surface strain $\gamma$, is given by

$$
\gamma=\frac{R}{d} \theta=F_{\gamma} \theta
$$

where $d$ is the height of the gap, and $\theta$ is the angular displacement of the rheometer. Values of $G^{\prime}$ and $G^{\prime \prime}$ can be converted to $G_{S}^{\prime}$ and $G_{S}^{\prime \prime}$ by multiplication by a factor of $F_{S \sigma} / F_{B \sigma}=R / 4$.

In the stiff state, calculated values of $G_{S}^{\prime}$ are consistent between the two plate sizes: the average elasticity for $\gamma_{0}<0.01$ is $G_{S}^{\prime}=13.3 \pm 2.6 \mathrm{~N} / \mathrm{m}$ with the $20 \mathrm{~mm}$ plate, and $G_{S}^{\prime}$ $=13.0 \pm 1.9 \mathrm{~N} / \mathrm{m}$ with the $40 \mathrm{~mm}$ plate. The reported errors are obtained from the standard deviation of the four measurements and are indicative of the significant variation in elasticity measurements, even from the same sample. Because $G_{S}^{\prime}$ measured in the stiff state is independent of the plate size, it is likely that the elasticity in this state originates only from the elastic properties of the oxide skin. In the "soft" state, values of $G_{S}^{\prime}$ are not consistent between the two plate sizes, as seen in Fig. 1(b). Nevertheless, the calculated values of $G_{S}^{\prime}$ in the apparent linear regime are still consistent with our original assumption that $\mathrm{Bo} \gg 1$,

$$
\mathrm{Bo}=\frac{\tau_{S}}{\tau_{B}}=\frac{3 \sigma_{S}}{R \sigma}=\frac{3}{R} \frac{G_{S}^{\prime} \gamma_{0}}{\eta \dot{\gamma}_{0}}=\frac{3}{R} \frac{G_{S}^{\prime}}{\eta \omega},
$$

where $\eta$ is the bulk viscosity, and $\dot{\gamma}_{0}=\gamma_{0} \omega$, where $\omega$ is the oscillation frequency. For our experiments, $\omega=1 \mathrm{rad} / \mathrm{s}, \eta=0.002 \mathrm{~Pa} \mathrm{~s}$, and $\mathrm{Bo} \approx 10^{4}$ for both plates in the stiff state. In the soft state, $\mathrm{Bo} \approx 10^{3}$ for the $40 \mathrm{~mm}$ plate and $10^{2}$ for the $20 \mathrm{~mm}$ plate. Large values of Bo are consistent with our expectations that the surface stresses are more important than the bulk stresses in our experiments.

\section{B. Steady and oscillatory shear}

The elastic characteristics of the oxide skin are highly dependent on the shear history. Because the shear-history dependence can change $G_{S}^{\prime}$ by more than an order of magnitude, it can potentially lead to large discrepancies between different measurements of the mechanical properties of the oxide skin. These strain history effects might be present in other configurations that are specifically designed to measure surface rheology. To control these effects during rheological measurements, it is important to understand the mechanisms that cause them. We seek to better understand the difference between the stiff and soft states, by first considering the conditions under which both states were measured. Analysis of the strain oscillations reveals that when the material is in the stiff state, significant internal stresses from the pre-shear are stored in the oxide film. These stresses vanish during the large oscillations and are not present in the soft state, suggesting that the internal stress plays an important role in determining $G_{S}^{\prime}$.

In the strain-controlled oscillations shown in Fig. 1, the stiff state is probed immediately after the pre-shear, with no time allowed for relaxation of the material stresses. 
(a)

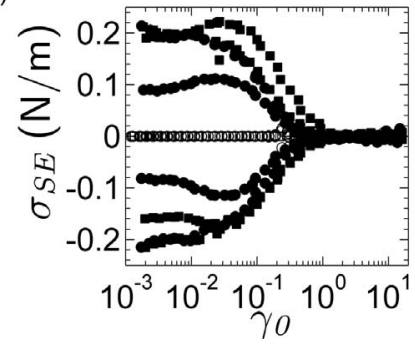

(b)

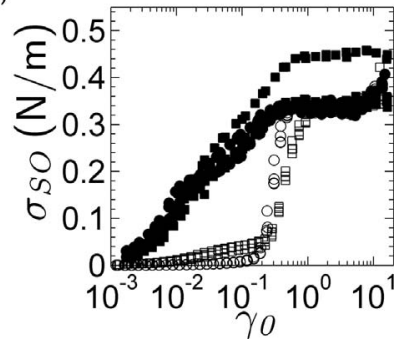

FIG. 2. (a) Steady $\sigma_{S E}$ and (b) oscillatory $\sigma_{S O}$ surface stress components calculated from strain-controlled oscillatory measurements on EGaIn performed immediately after steady pre-shears of $\pm 1.3 \mathrm{~s}^{-1}$ and $\pm 13 \mathrm{~s}^{-1}$. Ascending (closed) and descending (open) strain amplitude $\gamma_{0}$ sweeps performed with parallel plates of $20 \mathrm{~mm}$ (circles) and $40 \mathrm{~mm}$ (squares). Values of $\sigma_{S E}$ are nonzero in the stiff state, and the sign of $\sigma_{S E}$ matches that of the pre-shear. For $\gamma_{0}>0.5, \sigma_{S O}$ plateaus near $0.3 \mathrm{~N} / \mathrm{m}$ and $\sigma_{S E}=0$. One of the experiments with the $40 \mathrm{~mm}$ plate is an outlier and plateaus near $\sigma_{S O}=0.4 \mathrm{~N} / \mathrm{m}$.

These stresses tend to rotate the rheometer in the direction opposite to the direction of the pre-shear. The rheometer compensates for these stresses by providing a steady stress in the direction of the pre-shear, during the oscillatory tests. This is accomplished with a control loop that rapidly adjusts the applied torque to assure that the rheometer performs sinusoidal oscillations in strain about a fixed angular displacement. During the experiments, instantaneous torque and displacement values are provided by rheometer at a rate of 652 points per second. Using this data, we calculate both the steady and oscillatory components of $\sigma_{S}$. This is done by converting $\tau$ and $\theta$ values to $\sigma_{S}$ and $\gamma$ values, respectively, by using Eqs. (1) and (3). No corrections for bearing friction or inertia are necessary due to the low shear rates used. From these data, we obtain the maximum and minimum surface shear stresses $\sigma_{S}^{+}$and $\sigma_{S}^{-}$associated with each strain oscillation. In general, these stresses occur at the maximum and minimum strains, respectively. The average of the peak stress values is a measure of the steady, or even, stress $\sigma_{S E}$, and the difference is a measure of the oscillatory, or odd stress $\sigma_{S O}$,

$$
\begin{gathered}
\sigma_{S E}=\left(\sigma_{S}^{+}+\sigma_{S}^{-}\right) / 2, \\
\sigma_{S O}=\left(\sigma_{S}^{+}-\sigma_{S}^{-}\right) / 2 .
\end{gathered}
$$

When no internal sample stresses are present $\sigma_{S E}=0$. For the experiments shown in Fig. 1 , values of $\sigma_{S E}$ are nonzero because the oscillations are performed immediately after the pre-shear, with no sample relaxation. Values of $\sigma_{S E}$ have the same sign as the pre-shear, indicating that the rheometer is compensating for the tendency of the sample stresses to cause the rheometer to recoil. The steady stress $\sigma_{S E}$ remains constant for $\gamma_{0}<0.01$, where the material exhibits an elastic response, as shown in Fig. 2(a). In this regime $\left|\sigma_{S E}\right|$ $=0.14 \pm 0.07$ for the $20 \mathrm{~mm}$ plate, and $\left|\sigma_{S E}\right|=0.19 \pm 0.02$ for the $40 \mathrm{~mm}$ plate.

For $\gamma_{0}>0.01$, the rheological response begins to exhibit yielding, as indicated by the decrease in $G_{S}^{\prime}$ shown in Fig. 1. The yielding coincides with a gradual decrease in $\left|\sigma_{S E}\right|$, as shown in Fig. 2(a). Values of $\left|\sigma_{S E}\right|$ decrease until they plateau at zero, for $\gamma_{0}>0.5$. During the subsequent oscillations of descending $\gamma_{0}, \sigma_{S E}$ remains at zero for all $\gamma_{0}$, indicting that the material has lost its "memory" of the original pre-shear. This suggests that the yielding induced by the large oscillatory stresses may be responsible not only for lowering the apparent stiffness but also for removing the internal stresses that are imposed by the pre-shear. 
(a)

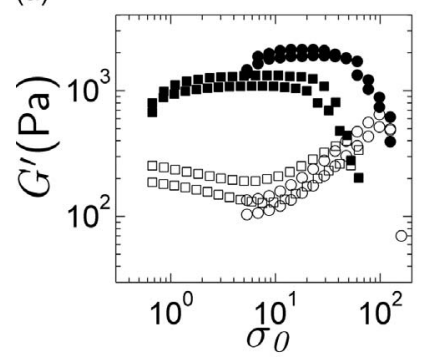

(b)

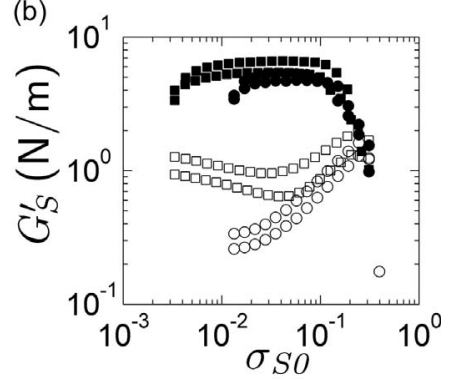

FIG. 3. Stress-controlled oscillatory measurements on EGaIn. Ascending (closed) and descending (open) strain amplitude sweeps performed with parallel plates of $20 \mathrm{~mm}$ (circles) and $40 \mathrm{~mm}$ (squares). (a) Values of the bulk modulus $G^{\prime}$ and the bulk stress amplitude $\sigma_{O}$ are not consistent between the two plate sizes. (b) The apparent surface modulus $G_{S}^{\prime}$ and the surface stress $\sigma_{S O}$ are more consistent between the two plate sizes.

In general, the oscillatory stresses $\sigma_{S O}$ are independent of the plate size, as shown in Fig. 2(b). At the onset of yielding, near $\gamma_{0}=0.01, \sigma_{S O} \approx 0.12 \mathrm{~N} / \mathrm{m}$. Values of $\sigma_{S O}$ increase with $\gamma_{0}$ until they roughly begin to plateau at $\sigma_{S Y}$, for $0.5<\gamma_{0}<13$ [Fig. 2(b)]. The development of the plateau in $\sigma_{S O}$ at a value of $\sigma_{S Y}$ coincides with the vanishing of $\left|\sigma_{S E}\right|$ [Fig. 2(b)]. As values of $\gamma_{0}$ subsequently descend, values of $\sigma_{S O}$ remain equal to plateau values until $\gamma_{0}$ descends below 0.5 . Here $\sigma_{S O}$ exhibits a sharp decline and then attains low values that correspond with the low values of $G_{S}^{\prime}$ that are measured in the soft state.

We calculate values of $\sigma_{S Y}$ by averaging $\sigma_{S O}$ over the plateau region from $3<\gamma_{0}$ $<13$, during both ascending and descending $\gamma_{0}$. Values of $\sigma_{S Y}$ were $0.36 \pm 0.03 \mathrm{~N} / \mathrm{m}$ with the $20 \mathrm{~mm}$ plate and $0.35 \pm 0.01 \mathrm{~N} / \mathrm{m}$ with the $40 \mathrm{~mm}$ plate. The similarity between the values obtained from the two plate sizes suggests that $\sigma_{S Y}$ originates from the oxide skin.

Values of $\sigma_{S Y}$ represent a limitation in the stress-carrying capability of the material. We expect that the measured values of $\sigma_{S Y}$ should be similar to values of $\sigma_{D Y}$ obtained from the microfluidic experiments, and which also represent a maximum in surface stress [Dickey et al. (2008)]. Our measured values of $\sigma_{S Y}$ are less than the value of $\sigma_{D Y}$ for EGaIn measured in atmosphere $(0.630 \mathrm{~N} / \mathrm{m})$. One possible reason for the difference is that the deformation in the microfluidic experiment is dilational as the meniscus extends into the channel, whereas in the parallel plate experiment, the deformation is dominated by shear.

Oscillations at the maximum stress $\sigma_{S Y}$ appear to be responsible for reducing $\left|\sigma_{\mathrm{SE}}\right|$ to zero, thereby giving rise to the soft state. Because $\left|\sigma_{S E}\right|=0$ in the soft state, and $\left|\sigma_{S E}\right|$ $\neq 0$ in the stiff state, it is reasonable to hypothesize that the soft state will be observed whenever $\left|\sigma_{S E}\right|=0$. This hypothesis is, however, discredited by performing stresscontrolled oscillations in which the rheometer provides a sinusoidal stress, so that $\left|\sigma_{S E}\right|$ $=0$. These experiments are performed on the same samples as the strain-controlled experiments and are preceded by unidirectional pre-shears of \pm 0.5 and $\pm 1 \mathrm{~s}^{-1}$ for the 20 and $40 \mathrm{~mm}$ parallel plates, respectively. We note that $G_{S}^{\prime}$ is are not very sensitive to the magnitude of the pre-shear. In these experiments, we still observe a clear distinction between the stiff and soft states, as shown in Fig. 3. As with the strain-controlled oscillations, bulk values of $G^{\prime}$ and $\sigma_{O}$ are not consistent between the two plate sizes [Fig. 3(a)] but are more consistent when rescaled to the surface values $G_{S}^{\prime}$ and $\sigma_{S O}$ [Fig. 3(b)]. The material exhibits a roughly linear elasticity for $\sigma_{S O}<0.1 \mathrm{~N} / \mathrm{m}$. The gradual increase in $G_{S}^{\prime}$ in the linear regime is most likely an effect of time because it occurs at different 


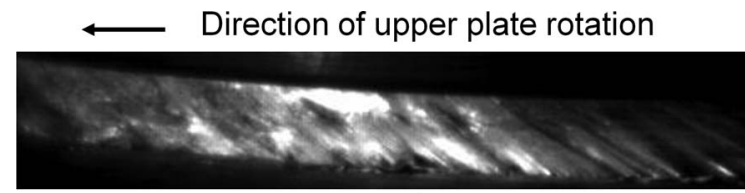

Stationary Lower Plate ${ }^{\prime}$

Direction of upper plate rotation

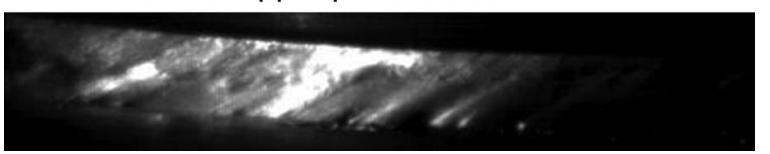

FIG. 4. Buckling or cracking in the direction of shear in EGaIn, in the AR-G2 rheometer. Buckling lines depend on the direction of rotation of the upper plate.

values of $\sigma_{S O}$. We estimate the linear elasticity of the stiff state from the maximum surface elasticity $G_{S M}^{\prime}$ from the near-linear regime. The average value of $G_{S M}^{\prime}$ from the two measurements performed is $6.0 \mathrm{~N} / \mathrm{m}$ for the $40 \mathrm{~mm}$ plate and $5.0 \mathrm{~N} / \mathrm{m}$ for the $20 \mathrm{~mm}$ plate. Despite the scatter associated with these measurements, values of $G_{S}^{\prime}$ are consistently about half of those measured using strain-controlled tests. This indicates that $G_{S}^{\prime}$ is an increasing function of the steady stress $\left|\sigma_{S E}\right|$. Nevertheless, simply removing the steady stress, such that $\left|\sigma_{S E}\right|=0$, does not cause the material to immediately revert to the soft state.

Although this set of experiments has not elucidated the mechanism responsible for the strain-history dependence, it has shown that $G_{S}^{\prime}$ is sensitive to the stress stored in the oxide film. This result implies that we can better understand the strain-history dependence of $G_{S}^{\prime}$ by studying how stress is stored in the oxide film, how it depends on the shear history, and how it depends on time.

\section{Surface visualization}

To better understand how the surface stress influences the deformation of the oxide skin, we imaged the sample while it was loaded in the parallel plate geometry. We used a Troubleshooter T-1000 (Fastec) camera fitted with a Nikon camera lens. When sheared, the oxide surface develops lines on the oxide surface that form at $45^{\circ}$ from the direction of shear and that span the height of the gap. When the direction of shear stress reverses, the lines re-orient in the new shear direction as shown in Fig. 4. These lines may indicate that there is shear-induced buckling or cracking of the film. Similar buckling lines are also observed in thin cylindrical shells buckled under torsion [Timoshenko (1936); Winterstetter and Schmidt (2002)]. When oscillations are sufficiently large to induce yielding, samples exhibit shear localization. When this occurs, the lower portion of the sample is stationary, and shear is localized in a region near the upper plate, where breakup of the oxide skin is apparent. These observations demonstrate the solid-like nature of the oxide interface. They also indicate that the deformation of the oxide skin in complicated and that the shape of the oxide film can change in response to the stress.

\section{Normal forces}

We enhance our ability to monitor the stress evolution in the oxide films by supplementing our measurements of surface stress with measurements of the normal force $f$. These measurements suggest that the surface stress of the oxide film affects the normal 
force in a way that is similar to how the surface tension of simple liquids affects normal force measurements in parallel plate experiments. Simple liquids in the parallel plate geometry typically exert a pressure $p$ on the bulk of the sample that tends to push the parallel plates away from each other, thereby giving rise to a positive normal force measurement. The relationship between $p$ and the surface tension $\gamma_{T}$ is given by

$$
p=\gamma_{T}\left(\frac{1}{R}-\frac{2 \cos \theta}{d}\right),
$$

where $\theta$ is the contact angle that the sample makes with the parallel plate, measured through the liquid phase [Hutton (1972)]. This relation can be easily derived by considering a vertical cross section that cuts the sample along the diameter of the parallel plates. The force on this plane due to the liquid pressure is balanced by the component of surface tension that acts normal to the plane, according to $p(2 R d)=\gamma_{T}(2 d-4 R \cos \theta)$. The pressure $p$ is then related to the normal force $f$, according to [Hutton (1972)],

$$
p=\frac{f}{\pi R^{2}} \text {. }
$$

Standard relations between $p$ and $\gamma_{T}$ in simple liquids can be applied to oxide-coated liquid metals. In our earlier experiments on flow in microchannels, we characterized oxide skins in terms of a maximum surface stress $\sigma_{D Y}$ that the material can support when it is subjected to dilation [Dickey et al. (2008)]. Measured values of $\sigma_{D Y}$ showed excellent agreement with apparent surface tensions measurements obtained with the pendant drop method. Due to the solid-like character of the oxide film, dilational surface stresses $\sigma_{D}<\sigma_{D Y}$ can be supported elastically by the oxide skin. In the parallel plate geometry, a surface dilational stress $\sigma_{D}$ exerts pressure on the bulk liquid. The relationship between $p$ and $\sigma_{D}$ is obtained with the same force balance that is used to obtain Eq. (6), with $\sigma_{D}$ playing the role of $\gamma_{T}$,

$$
p=\sigma_{D}\left(\frac{1}{R}-\frac{2 \cos \theta}{d}\right) .
$$

This relationship predicts that measurements of $p$ allow us to directly probe the stress state of the oxide film. Here we validate this prediction by demonstrating a direct correlation between $p$ and $\sigma_{S}$.

Our normal force measurements are performed using the AR-G2 because normal force measurements obtained with this instrument are repeatable, whereas those obtained using the Malvern rheometers are not. Normal force measurements in the AR-G2 are obtained with a strain gauge located underneath the bottom plate. This gauge measures the normal force $f$ exerted by the sample on the lower plate of the rheometer. We then convert $f$ to the pressure $p$ exerted on the plates according to Eq. (7). Positive values of $p$ correspond to downward forces on the bottom plate.

Most of the effects that we demonstrate have been reproduced in both EGaIn and Ga, indicating that they are not material specific. Both materials feature a skin composed of oxides of Ga [Dickey et al. (2008)]. The experiments we present in the remainder of the paper were obtained with Ga.

As with $G_{S}^{\prime}$, measurements of $p$ are highly dependent on shear history. The initial shear history of a given sample is not precisely known because the samples are submitted to constant shear during sample loading. For this reason, it is difficult to establish the zero point of the measurements of $p$. For convenience, we assign $p=0$ to be the value measured at the onset of the first oscillatory measurement. Although this point is arbitrary, we 
(a)
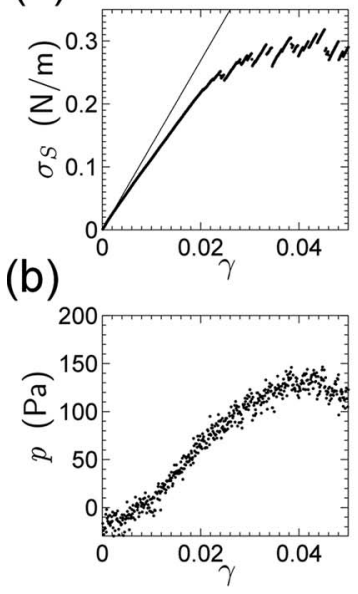

(c)

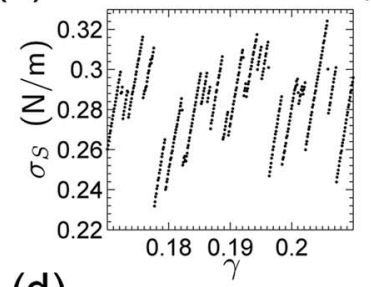

(d)

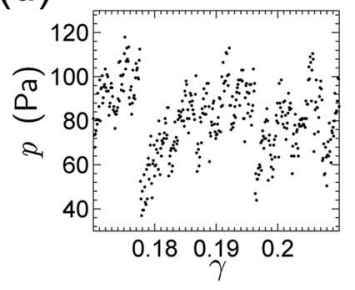

(e)

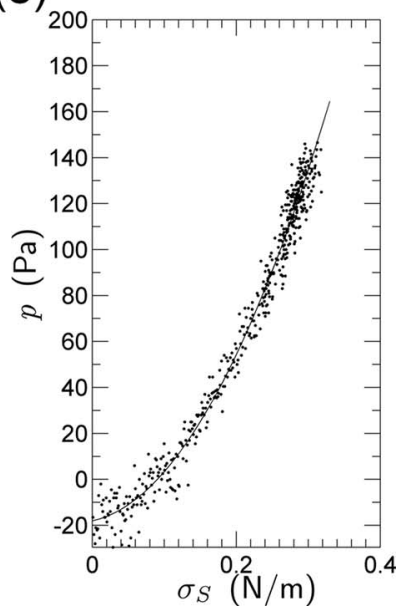

FIG. 5. Strain ramp experiments on Ga. The strain is increased at a rate of $10^{-4} \mathrm{~s}^{-1}$. (a) $\sigma_{S}$ and (b) $p$ increase with $\gamma$ for $\gamma<0.04$ and plateau for $\gamma>0.04$. (c) Within the plateau region, values of $\sigma_{S}$ exhibit increases that are punctuated by sharp drops, probably indicating stick/slip behavior. (d) Similar patterns are apparent in values of $p$. (e) The non-linear dependence of $p$ on $\sigma_{S}$ is shown for $0<\gamma<0.5$. The line is a parabolic fit to the data, and is a guide to the eye.

use this reference for all measurements of $p$ that we present in this paper, which were obtained from a single sample. We also checked that the axial compliance of the rheometer is sufficiently high that the normal forces we observe should have virtually no effect on the gap height. We also perform all measurements at sufficiently low strain rates that any spurious normal force effects due to inertia are negligible [Walters (1975)].

We probe the dependence of $p$ on $\sigma_{S}$ by performing two simple experiments: a strain ramp test and a stress relaxation test. Because the results of our experiments are highly dependent on strain history, it is important to precede the experiments with a standardized pre-shear routine that can consistently move the material into a reproducible state. Our experiments with the HRnano rheometer demonstrate that this is achievable by performing a unidirectional pre-shear that reproducibly moves the material into the stiff state. The unidirectional pre-shear therefore forms the basis of an initialization routine that we perform before all measurements. This routine consists of a pre-shear of $1 \mathrm{~s}^{-1}$ for $30 \mathrm{~s}$, followed by a stress equilibration period of $5 \mathrm{~min}$, in which no stress is applied.

Following this initialization, we perform the strain ramp test by increasing the strain at a rate of $10^{-4} \mathrm{~s}^{-1}$, while collecting $\sigma_{S}$ and $p$ measurements at a rate of 1 point per second. During the early part of the strain ramp, $\sigma_{S}$ increases linearly with strain but deviates from linearity as the strain increases, as shown in Fig. 5(a). From the fit of a line to the first ten data points $(\gamma<0.001)$, we obtain an apparent shear modulus of $G_{S}^{\prime}$ $=13.5 \mathrm{~N} / \mathrm{m}$. Values of $p$ were nearly constant at small strains but begin to increase at about $\gamma=0.005$, as shown in Fig. 5(b).

Both $\sigma_{S}$ and $p$ plateau for $\gamma>0.04$, as shown in Figs. 5(a) and 5(b). The plateau value of $\sigma_{S}$ is about $0.3 \mathrm{~N} / \mathrm{m}$. A close look at the plateau region reveals short periods of increases in $\sigma_{S}$, punctuated by discontinuous drops in $\sigma_{S}$ [Fig. 5(c)]. This likely indicates stick-slip flow. The increases in $\sigma_{S}$ likely correspond to sample adhesion to the rheometer plates, leading to increased sample strain, and the sudden drops in $\sigma_{S}$ likely correspond to slip events, where the strain is localized in a thin region near the plate. This localized flow allows the oxide skin to "slip" back to a configuration of lower strain, thereby 
(a)

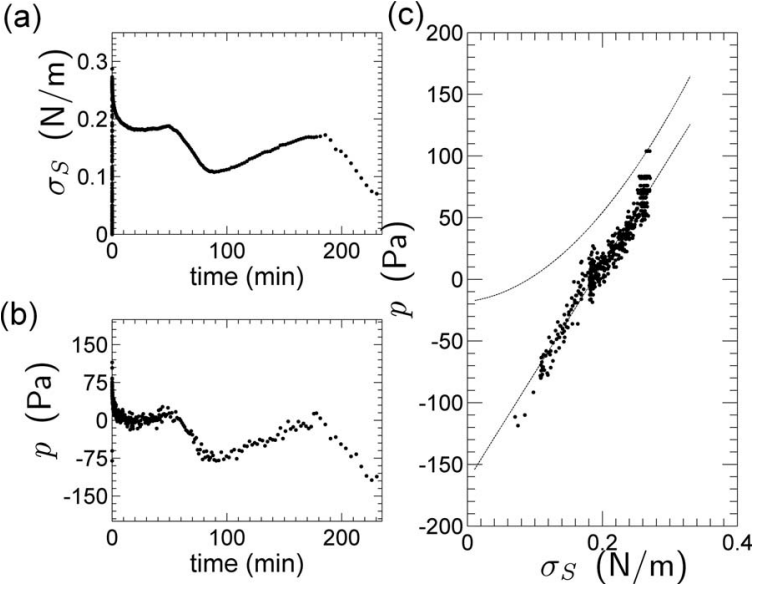

FIG. 6. Stress relaxation experiment performed on Ga. After flowing in the stick/slip regime, the sample is held at a constant $\gamma$ while (a) $\sigma_{S}$ and (b) $p$ evolve. Sometimes an unusual increase in both $\sigma_{S}$ and $p$ is observed. (c) Nevertheless, the $p$ remains roughly proportional to $\sigma_{S}$ at all times, as shown by a linear fit (straight line). The parabolic line in (c) is the same parabola that is shown in Fig. 5(e).

quickly relaxing the stress that accrued during the stick events. Although the normal stress data are noisy, they appear to increase with $\sigma_{S}$ during the stick events and to decrease rapidly with $\sigma_{S}$ during the slip events [Fig. 5(d)]. These observations are consistent with our optical observations of localized flow. In the flow, or slip, regime the average $\sigma_{S}$ required to rotate the upper plate at a constant angular velocity, or apparent shear rate, depends only weakly on the rate (usually decreasing with increasing shear rate) and was about $0.2-0.3 \mathrm{~N} / \mathrm{m}$ for the Ga sample shown here [Dickey et al. (2008)]. The interdependence of $p$ and $\sigma_{S}$ is shown by a plot of $p$ as a function of $\sigma_{S}$ in the region $0<\gamma<0.05$, as indicated by Fig. 5(e).

The correlation between $p$ and $\sigma_{S}$ is further demonstrated in a stress relaxation test. This experiment was performed by ramping the strain from $\gamma=0$ to $\gamma=1$ in $10 \mathrm{~s}$. This deformation was sufficiently large to move the material well into the flow or slip regime. The rheometer then maintained the upper plate at the angular displacement obtained at the end of the strain ramp for several hours. During this time period, the rheometer recorded the $\sigma_{S}$ values required to maintain this displacement, as well as $p$ measurements. During the experiment, both $\sigma_{S}$ and $p$ decreased from their peak values over time scales of several hours, as observed in Figs. 6(a) and 6(b). During the relaxation, an unusual effect is observed in some samples: the downward trend is interrupted by periods of increasing $p$ and $\sigma_{S}$. A plot of $p$ versus $\sigma_{S}$ shows that during both ascending and descending regions, these two measurements remain roughly proportional. A linear fit to $p$ as a function of $\sigma_{S}$ reveals a slope or proportionality constant of $875 \mathrm{~m}^{-1}$ and a $y$-intercept of $-163 \mathrm{~Pa}$. The value $p=-163 \mathrm{~Pa}$ may therefore represent the true state of zero pressure. The unusual and temporary increase with time in both $p$ and $\sigma_{S}$ and $p$ were reproduced on both the AR-G2 with Ga and the HRnano with EGaIn.

It is possible to visualize a mechanism that may be responsible for the correlation between $p$ and $\sigma_{S}$ by considering a thin elastic string with each of its ends attached to the outer rims of two concentric parallel circles, or plates, and spanning the gap between the plates. The distance between the parallel plates is fixed, as is nearly the case with the parallel plates of our experiment. The minimum stretching of the elastic "string" occurs when the plates are rotated such that the string is perpendicular to the plates. Any rotation 


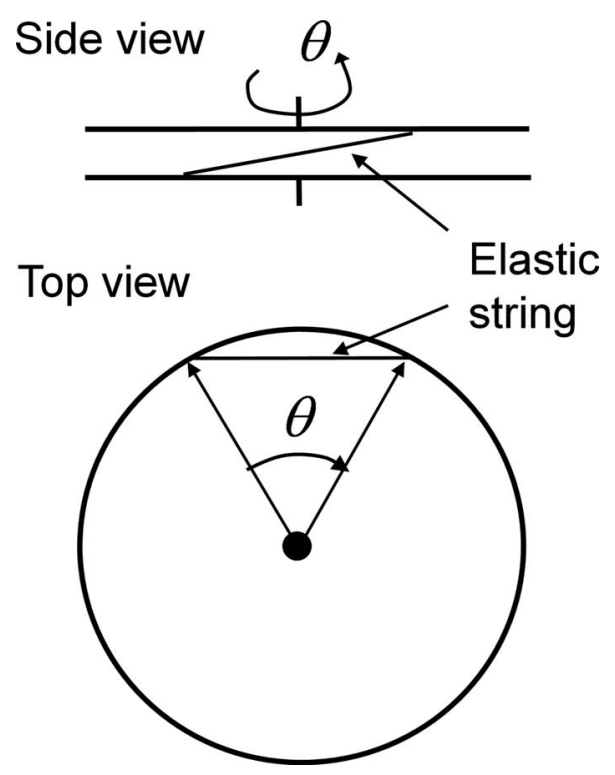

FIG. 7. An elastic string spanning the gap between two parallel plates at fixed separation. When one plate is rotated relative to the other through an angle $\theta$, the projection of the stretched spring forms a chord on the circle corresponding to the plate.

of the plates away from this point of "zero strain" will cause the elastic string to stretch. The string will minimize its stretching by tracing the path of shortest distance between the attachment points of the string. If this path is projected onto one of the plates, it will not follow the arc of the circles but will be a chord (see Fig. 7). If the same parallel plates are now connected by a thin elastic membrane attached to the outer rims of the plates, then relative rotation of the plates will cause the membrane to stretch. If the stretched elastic elements of the membrane are modeled as a series of elastic strings regularly spaced around the circumference of the plates, then upon shearing, the strings will trace the path of shortest distance between their end points and the membrane will bow inward.

The tendency of the membrane to bow inward when sheared tends to decrease the volume enclosed by the membrane and the parallel plates. However, if this space is occupied by an incompressible fluid, then the fluid can accommodate the bowing of the outer membrane by slightly displacing the parallel plates away from each other. In our experiments, this displacement is detected by the strain gauges. The relaxation of the tension in the elastic strings is accompanied by a decrease in the measured pressure $p$ of the bulk.

The stress relaxation experiment is similar to the strain-controlled oscillations that we performed using two different plate size with the HRnano. The oscillations were performed immediately following a pre-shear and without allowing the material to relax. In the oscillatory experiments, the steady stress $\sigma_{S E}$ vanished when $\gamma_{0}$ became sufficiently large, which occurred after about $15 \mathrm{~min}$ of oscillations. However, in the stress relaxation experiment in which no oscillations were performed, the steady stress persisted for several hours, in both Ga and EGaIn. This result indicates that the large oscillations do indeed play an essential role in causing $\sigma_{S E}$ to vanish during the oscillatory tests.

The experiments performed on the HRnano also showed that surface stresses play a 

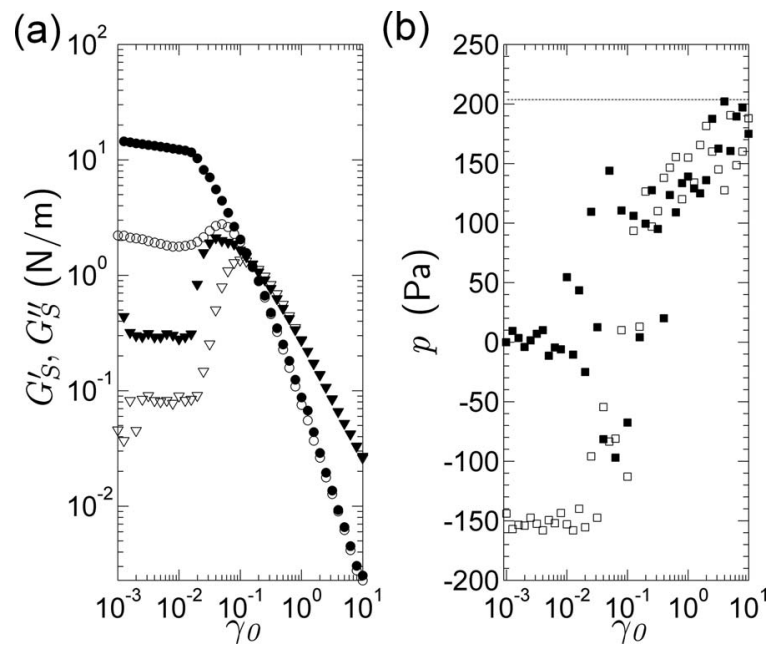

FIG. 8. Strain amplitude sweep on Ga (experiment no. 1). (a) Surface viscoelastic moduli $G_{S}^{\prime}$ (circles) and $G_{S}^{\prime \prime}$ (triangles), and (b) $p$, measured during strain sweeps of ascending (closed symbols) and descending (open symbols) $\gamma_{0}$. The horizontal dashed line in (b) is the average $p$ measured during the pre-shear.

role in enhancing the surface elasticity in the stiff state. We therefore hypothesize that the stiff state is associated with elevated values of $p$. Additional oscillatory experiments with the AR-G2 demonstrate that this is indeed the case.

We preceded our first oscillatory experiment with the same standard pre-shear routine that was performed before the strain-ramp experiment: the stiff state is induced by imposing a constant strain rate of $1 \mathrm{~s}^{-1}$ for $30 \mathrm{~s}$, and then the sample is allowed to equilibrate for $5 \mathrm{~min}$, with no stress applied. The viscoelastic properties of the sample are then probed with strain-controlled oscillations of both ascending and descending strain amplitude $\gamma_{0}$. Although the $5 \mathrm{~min}$ equilibration should, in principle, allow stress relaxation to occur, our sample clearly exhibits stiff and soft states during the oscillations of both ascending and descending $\gamma_{0}$, respectively, as shown in Fig. 8(a).

During the oscillatory measurements, the rheometer provides a single value of $p$ corresponding to each strain oscillation. The value of $p$ measured during the first oscillation of the first oscillatory experiment is taken to be $p=0$. At low $\gamma_{0}, p$ is independent of $\gamma_{0}$. As $\gamma_{0}$ increases above the yield strain, $\gamma_{Y}=0.02, p$ becomes an increasing function of $\gamma_{0}$, as shown in Fig. 8(b). For $\gamma_{0} \approx \gamma_{Y}$, values of $p$ exhibit considerable scatter, most likely because $p$ changes substantially with $\gamma$ in the range $0<\gamma<\gamma_{Y}$, as demonstrated in the strain ramp experiments. As $\gamma_{0}$ becomes large relative to $\gamma_{Y}$, we expect a greater portion of the strain oscillation to be dominated by the high- $p$ plateau observed in the strain ramp experiment. It is therefore not surprising that as $\gamma_{0}$ increases beyond $\gamma_{Y}$, the scatter of the $p$ measurements decreases. In this limit, values of $p$ are similar to those measured during the pre-shear shown by the dotted line in Fig. 8(b). As $\gamma_{0}$ subsequently decreases, $p$ also decreases, eventually reaching a plateau at about $-150 \mathrm{~Pa}$ in the soft state. The stiff and soft states are therefore not only distinguished by large and small values of $G_{S}^{\prime}$, respectively, but also by large and small values of $p$.

The positive correlation between $G_{S}^{\prime}$ and $p$ is reproduced in a series of oscillatory measurements, in which we varied several experimental parameters. In these experiments, the conditions of the first oscillatory experiment (experiment no. 1, shown in Fig. 8) correspond to the standard experiment. In the subsequent experiments, we varied one 
(a)

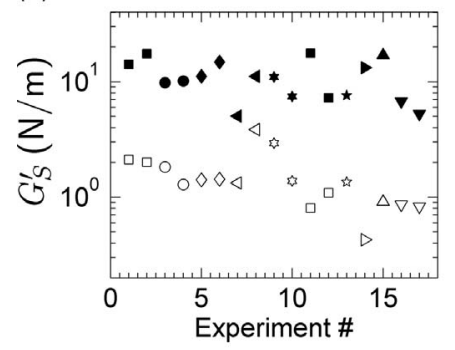

(b)

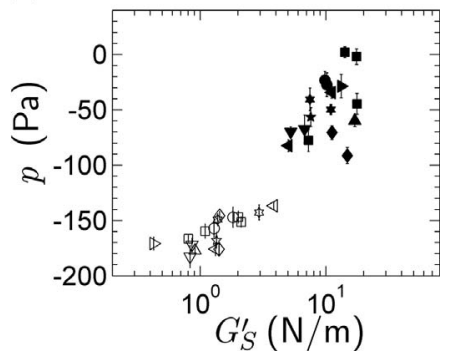

FIG. 9. Measurements of $G_{S}^{\prime}$ from Ga, in both the stiff (closed symbols) and soft (open symbols) states under a variety of experimental conditions. The initial experiment represents a reference experiment (ם): pre-shear: $\pm 1 \mathrm{~s}^{-1}$, equilibrium time: $5 \mathrm{~min}$, rate-controlled oscillations, and oscillation frequency: $1 \mathrm{rad} / \mathrm{s}$. In each subsequent experiment, we varied one or two of the four parameters of the reference experiment, including: pre-shear: $\pm 10 \mathrm{~s}^{-1}(\boldsymbol{\bullet})$, equilibrium time: $20 \mathrm{~s}(\boldsymbol{\nabla}), 20 \mathrm{~min}(\boldsymbol{\nabla})$, and $1 \mathrm{~h}(\boldsymbol{\Delta})$. Stress-controlled $(\boldsymbol{\Delta})$. Stresscontrolled and $\pm 10 \mathrm{~s}^{-1}(*)$. Oscillation frequency: $4 \mathrm{rad} / \mathrm{s}(\star)$ and $0.25 \mathrm{rad} / \mathrm{s}(\boldsymbol{)})$. (a) Measured values of both the stiff and soft states vary little from experiment to experiment. (b) The pressure $p$ is an increasing function of $G_{S}^{\prime}$.

or two of the standard experiment parameters (with the "standard" condition shown in bold): the magnitude of the pre-shear $\left( \pm 1\right.$ and $\left.\pm 10 \mathrm{~s}^{-1}\right)$, the equilibrium time $(20 \mathrm{~s}$, $5 \mathrm{~min}, 20 \mathrm{~min}$, and $1 \mathrm{~h}$ ), rate-controlled vs stress-controlled, and the oscillation frequency $(4, \mathbf{1}$, and $0.25 \mathrm{rad} / \mathrm{s})$. We obtained average values of $G_{S}^{\prime}$ and $p$ in the linear regime, by averaging these values over the five oscillatory measurements of lowest strain. These oscillatory experiments, together with additional experiments, including the strain ramp and stress relaxation experiments shown previously, were performed over approximately $30 \mathrm{~h}$ on the same sample. As with the results obtained with the HRnano, the sample can change between the stiff and soft states reversibly, as shown in plots of $G_{S}^{\prime}$ from each experiment, in Fig. 9(a). Values of $G_{S}^{\prime}$ measured in the stiff and soft states are fairly stable in time, or perhaps decrease slightly in time. Nevertheless, any timedependence of $G_{S}^{\prime}$ is less significant that the different between the stiff and soft states. For all experimental conditions, values of $p$ are consistently higher in the stiff state [solid symbols, Fig. 9(b)] than in the soft state (open symbols). Values of $p$ measured in the soft state are typically close to the value of $p=-163 \mathrm{~Pa}$, estimated from the stress relaxation test to be the pressure corresponding to $\sigma_{S}=0$. This observation agrees with our result from the HRnano that the soft state corresponds to a vanishing of the stresses in the oxide film and that the stiff state is associated with elevated stresses in the oxide film.

In general, the parameters that we vary do not appear to have a significant effect on the elasticity measurements. However, within the $G_{S}^{\prime}-p$ space shown in Fig. 9(b), the stiffstate measurements at high $G_{S}^{\prime}(\approx 15 \mathrm{~N} / \mathrm{m})$ and low $p(\approx-100 \mathrm{~Pa})$ are occupied by the measurements of longest wait time, including $1 \mathrm{~h}$ (experiment no. 15, $\mathbf{\Delta}$ ) and $20 \mathrm{~min}$ (experiments nos. 5 and $6, \diamond$ ). This implies that $p$ decreases with time during the equilibrium period. This may be associated with a relaxation of the stresses associated with the stiff state. Because the soft state is associated with low values of $p$, it is reasonable to expect that a decrease in $p$ values during the equilibrium time should correspond to a decrease in $G_{S}^{\prime}$. We would therefore expect lower values of $G_{S}^{\prime}$ to be observed after the longest equilibrium times. However, in the data we observe opposite trends: some of the lowest values of $G_{S}^{\prime}$ (experiments nos. 16 and 17, $\boldsymbol{\nabla}$ ) were measured in experiments of shortest equilibrium time $(20 \mathrm{~s})$, and one of the highest values of $G_{S}^{\prime}$ (experiment no. 15, $\mathbf{\Delta}$ ) was obtained after the longest equilibrium time $(1 \mathrm{~h})$. This implies that $G_{S}^{\prime}$ may be an increasing function of the equilibrium time. These observations led us 


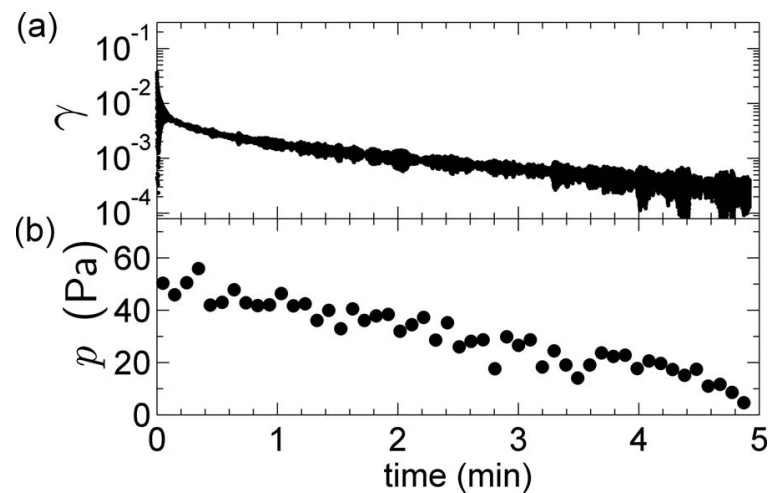

FIG. 10. Equilibrium period following the $30 \mathrm{~s}$ pre-shear of $1 \mathrm{~s}^{-1}$ on Ga (experiment no. 1). (a) Because the pre-shear was in the positive direction, $\gamma$ values gradually decrease during the equilibrium period, indicating that stresses that were stored during the pre-shear are relaxing. (b) The stress relaxation corresponds to a decrease in $p$ through the equilibrium period.

to perform additional analysis, which showed that during the equilibration period, $p$ decreases and $G_{S}^{\prime}$ increases. This analysis confirms that the apparent properties of the oxide skin are sensitive to time.

\section{E. Time-dependence}

Our analysis is performed on the raw angular displacement and normal force data that are provided by the rheometer during the equilibration period at a rate of 250 data points per second. We convert these values to $\gamma$ and $p$, respectively, and observe that immediately following the cessation of the pre-shear, the rheometer reverses direction and exhibits damped ringing about a new equilibrium position. The ringing is due to a combination of sample elasticity and rheometer inertia, and the damping occurs because of viscous dissipation in the sample. After the oscillations damp out, the rheometer slowly drifts in the direction opposite of the pre-shear. In the case of experiment no. 1 (corresponding to the oscillations shown in Fig. 8), values of strain steadily decrease because the pre-shear had been applied in the positive direction [see Fig. 10(a)]. In experiments in which the pre-shear was in the negative direction, the values of strain subsequently increase during the equilibrium period. The slow elastic recoil that follows the pre-shear persists for the entire equilibration period, even when the equilibration is as long as $1 \mathrm{~h}$. The recoil indicates that the elastic stresses that were stored during the preceding preshear are slowly relaxing. Because $p$ is an increasing function of $\sigma_{S}$, we expect that the relaxation of $\sigma_{S}$ should correspond to a decrease in $p$. We test this hypothesis by binning the raw measurements of $p$ obtained from the rheometer into increments that are linearly spaced in time and averaging all the $p$ values within each bin. We then plot the average $p$ value from each bin as a function of the average time of each bin. These plots show a gradual decrease in $p$ during the equilibrium period, as shown for experiment no. 1 in Fig. 10(b). The decrease in $p$ persists throughout the equilibration period, even when it lasts for as long as $1 \mathrm{~h}$.

Because the surface stresses decay slowly, we hypothesize that residual steady stresses are present during the strain-controlled oscillatory measurements performed after the equilibration period. We test for steady stresses by calculating both the steady and oscillatory shear stress components $\sigma_{S E}$ and $\sigma_{S O}$, from the strain-controlled oscillations using the same method that we used to analyze the data from the HRnano: raw torque values 
(a)

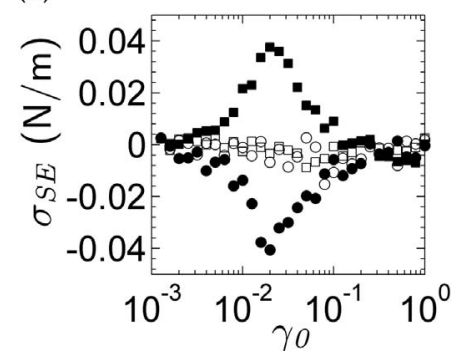

(b)

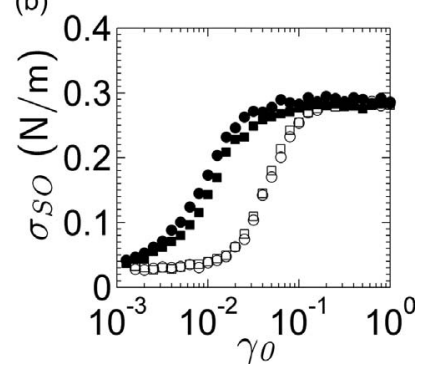

FIG. 11. (a) Steady $\sigma_{S E}$ and (b) oscillatory $\sigma_{S O}$ stress components from experiment nos. 1 and 2 performed on $\mathrm{Ga}$. The strain oscillations were performed at $1 \mathrm{rad} / \mathrm{s}$ during both ascending $\gamma_{0}$ (closed symbols) and descending $\gamma_{0}$ (open symbols). The tests were performed after pre-shears of $1 \mathrm{~s}^{-1}$ (experiment no. 1, squares) and $-1 \mathrm{~s}^{-1}$ (experiment no. 2, circles). The sign of $\sigma_{S E}$ matches the sign of the pre-shear.

obtained during the oscillations were used to calculate $\sigma_{S}$ values during the oscillations. Peak $\sigma_{S}$ values from each oscillation were then used to calculate $\sigma_{S E}$ and $\sigma_{S O}$. No corrections for bearing friction or inertia were necessary due to the low shear rates used. In agreement with our prediction, these data show that in the stiff state following the equilibrium, $\sigma_{S E} \neq 0$ and possesses the same sign as the direction of the pre-shear: this is because after a pre-shear in the positive direction, the rheometer must provide a steady stress $\sigma_{S E}$ in the positive direction to prevent elastic recoil. This result is shown for experiment nos. 1 and 2, where the applied pre-shears are $\pm 1 \mathrm{~s}^{-1}$, respectively [Fig. 11(a)]. Interestingly, during the strain oscillations, $\sigma_{S E}$ exhibits a maximum at $\gamma_{0}=\gamma_{Y}$ $=0.02$. Moreover, in agreement with the data obtained using the HRnano, $\sigma_{S E}=0$ during the oscillations of descending $\gamma_{0}$ where the soft state is apparent. Our observations show that residual stresses persist throughout the equilibration period and only vanish when the material is in the soft state. Values of $G_{S}^{\prime}$ also remain elevated throughout the equilibrium period and only vanish when the material is in the soft state.

To understand the time-dependence of $G_{S}^{\prime}$ and $G_{S}^{\prime \prime}$, we calculate these values throughout the equilibration period by analyzing free inertio-elastic oscillations. Free oscillations during the first $5 \mathrm{~s}$ of the equilibration period of experiment no. 1 are shown in Fig. 12(a). These oscillations occur as a result of both rheometer inertia and sample elasticity, and

(a)

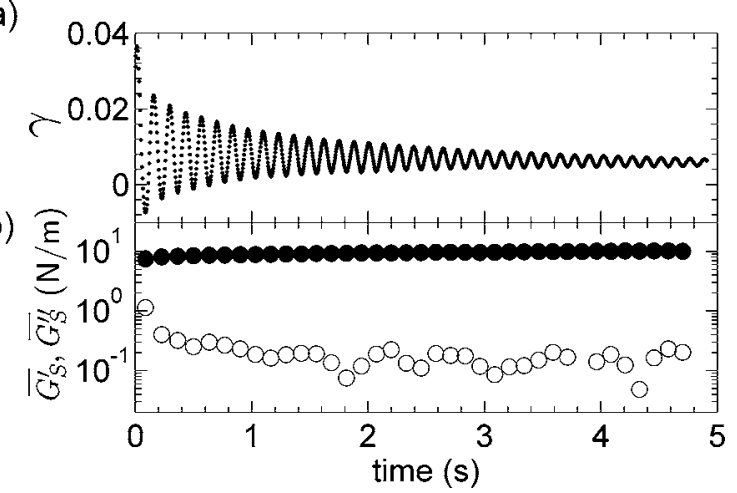

FIG. 12. (a) Strain oscillations in Ga immediately after the pre-shear in experiment no. 1. (b) $G_{S}^{\prime}$ (closed symbols) and $G_{S}^{\prime \prime}$ (open symbols) calculated from the oscillations. 

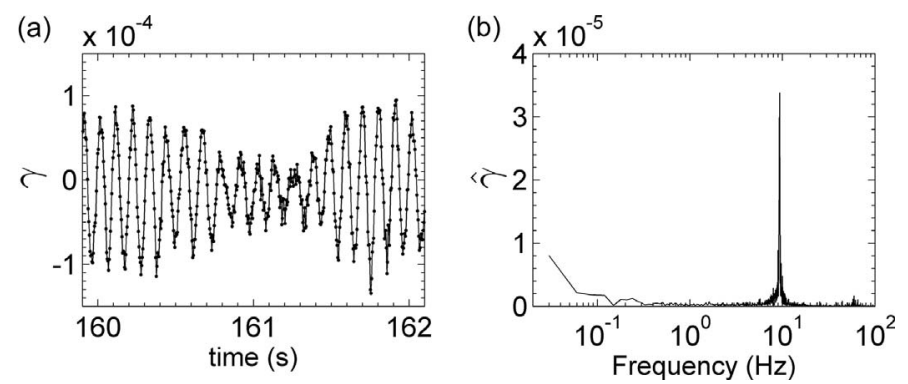

FIG. 13. (a) Detail of strain oscillations in Ga during the equilibrium period of experiment no. 1. (b) A typical FFT of strain oscillations between 127 and $194 \mathrm{~s}$ following the pre-shear of experiment no. 1 .

they are damped by viscous dissipation in the sample. Analysis of oscillations therefore yields information about both the elastic and viscous properties of the sample. We analyze this data using the standard equations

$$
\begin{gathered}
G_{S}^{\prime} \approx \frac{I \omega_{0}^{2}}{b}\left(1+\left(\frac{\Delta}{2 \pi}\right)^{2}\right)=\bar{G}_{S}^{\prime}, \\
G_{S}^{\prime \prime} \approx \frac{I \omega_{0}^{2}}{b}\left(\frac{\Delta}{\pi}\right)=\bar{G}_{S}^{\prime \prime},
\end{gathered}
$$

where $I$ is the moment of inertia of the rotating portion of the rheometer, including the bearing, spindle, and the upper plate, $\omega_{0}$ is the frequency of oscillation, $b=F_{S \gamma} / F_{S \sigma}$, and $\Delta$ is the logarithmic decrement defined as $\Delta=\ln \left(A_{n} / A_{n+1}\right)$, where $A_{n}$ is the amplitude of the $n$th peak relative to the equilibrium position [Struik (1967); Ewoldt and McKinley (2007)]. We obtained values of $\omega_{0}$ and $\Delta$ from the oscillations shown in Fig. 12(a), by first fitting the strain values in the region of each extrema to a parabola, and then calculating the extrema of the fit parabolas. We took $A_{n}$ to be the difference between neighboring extrema (a maximum and a minimum), and we took $\omega_{0}$ to be the inverse of the time between every second extrema. For each pair of neighboring extrema, we calculate values for $\bar{G}_{S}^{\prime}$ and $\bar{G}_{S}^{\prime \prime}$ by comparing it to the following pair of extrema. This analysis indicates that during the time period analyzed (nearly $5 \mathrm{~s}$ ), $\bar{G}_{S}^{\prime}$ increases slightly and $\bar{G}_{S}^{\prime \prime}$ decreases slightly, suggesting that the material becomes slightly more solid-like in the seconds following the pre-shear [Fig. 12(b)]. This is consistent with a time-dependent increase in $\bar{G}_{S}^{\prime}$ that was measured during stress-controlled oscillations performed immediately following the pre-shear, with the HRnano (Fig. 3)

Free oscillations typically damp out until the material effectively arrests at an equilibrium strain. However, a static equilibrium strain was not observed in our samples. At times greater than about $5 \mathrm{~s}$, the oscillations did not damp out completely but rather continued to vibrate throughout the equilibration time at a small amplitude that varied in time. At times, the amplitude of the vibrations gradually increased, and at other times it gradually decreased, as is apparent in Fig. 10(a), and in detail in Fig. 13(a). These small oscillations occur because the sample exhibits a combination of low viscosity and high elasticity that makes it sensitive to minor perturbations from either the environment or the instrument itself. The minor perturbations were not unique to the AR-G2; similar oscillations were also observed with the HRnano. During the small vibrations, a distinctive frequency is visually apparent, as shown in Fig. 13(a). We measure this frequency by computing a fast Fourier transform (FFT) $\hat{\gamma}$. A typical FFT is shown in Fig. 13(b). This 
(a)

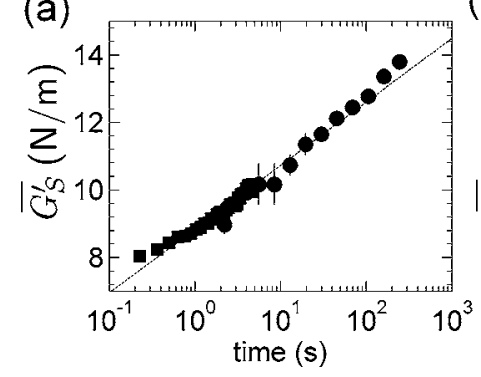

(b)

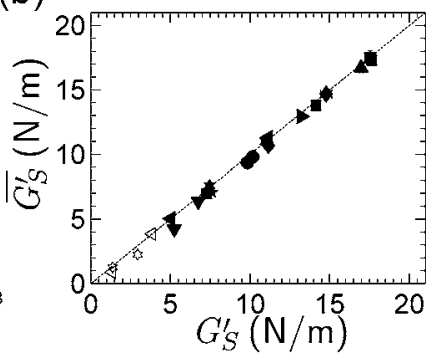

FIG. 14. Measurements of apparent surface elasticity $\bar{G}_{S}^{\prime}$ obtained from free strain vibrations of Ga. (a) Dependence of $\bar{G}_{S}^{\prime}$ on time during the stress equilibrium of experiment no. 1. Square symbols are obtained from the damped oscillations immediately following the pre-shear $t$ (also shown in Fig. 12). Circles are obtained from the FFT of small strain oscillations that are associated with the noise of the instrument. (b) Comparison between $\bar{G}_{S}^{\prime}$, obtained from FFTs of strain vibrations, and $G_{S}^{\prime}$ obtained from forced oscillations. Closed symbols correspond to the stiff state, and open symbols correspond to the soft state. The symbols correspond to the same experiments shown in Fig. 9. The dotted line represents $\bar{G}_{S}^{\prime}=G_{S}^{\prime}$.

FFT was calculated on strain data collected between 127 and 194 s following the preshear of experiment no. 1. Each FFT from the equilibrium period exhibits a strong maximum at frequency $\omega_{p}$. From this value, we calculate $\bar{G}_{S}^{\prime}=I \omega_{p}^{2} / b$ using Eq. (9a) and neglecting the viscous dissipation.

To monitor how $\bar{G}_{S}^{\prime}$ changes during the equilibrium time, we bin the strain data into time increments, such that the duration $\delta_{i}$, of the $i$ th increment is given by $\delta_{i}=t_{i+1}-t_{i}$. Values of $t_{i}$ are spaced logarithmically because the trends in our results are most apparent with plotted against the logarithm of time. Within each time period, we first fit the strain data to straight line, and then subtract the linear $\gamma$ contribution from the signal. This compensates for the drift shown in Fig. 10(a) and removes spurious low-frequency peaks in the FFT. Values of $\bar{G}_{S}^{\prime}$ calculated from each time period are plotted against the average time associated with that time period. At short times, the increments contain fewer data points, and there is greater uncertainty associated with $\omega_{p}$. Nine time increments at the earliest times were grouped into a single time increment, so that this composite time increment would contain enough data points for an accurate FFT.

Calculated values of $\bar{G}_{S}^{\prime}$ increase throughout the equilibrium time, as shown by the solid circles in Fig. 14(a). Values of $\bar{G}_{S}^{\prime}$ calculated from the FFT are similar to those calculated from the damped oscillations, as shown by the solid squares in Fig. 14(a). When both measurements are plotted as a function of the natural logarithm of time, they form a continuous line. We therefore quantify the dependence of $\bar{G}_{S}^{\prime}$ on time $t$ by fitting data from both measurements to the equation

$$
\bar{G}_{S}^{\prime}=\bar{G}_{S 0}^{\prime}+k \ln \left(t / t_{0}\right)
$$

where we assign $t_{0}=1$ s. For experiment no. $1, k=0.82 \mathrm{~N} / \mathrm{m}$ and $\bar{G}_{S 0}^{\prime}=8.8 \mathrm{~N} / \mathrm{m}$.

Similar trends were observed during the stress equilibrium periods of all of the oscillatory experiments shown in Fig. 9. However, in some tests there was a decrease in values of $\bar{G}_{S}^{\prime}$ during the first 2 or $3 \mathrm{~s}$ following the pre-shear, followed by a sustained increase in $\bar{G}_{S}^{\prime}$ with the logarithm of time. When it occurred, this decrease was not included in fits used to obtain $k$ and $\bar{G}_{S 0}^{\prime}$. Fits were performed to data that was processed using the same 
binning algorithms described for experiment no. 1, however, when the equilibration period is $20 \mathrm{~s}$, where the time increments used for the FFT measurements are spaced linearly. The average $k$ from all experiments is 0.65 and the standard deviation is 0.23 , indicating considerable variation. The mean and standard deviation values of $\bar{G}_{S 0}^{\prime}$ are 6.7 and $2.5 \mathrm{~N} / \mathrm{m}$, respectively. Because of the time-dependent increase in elasticity during the equilibrium period, these values are less than values of $G_{S}^{\prime}$ obtained by performing straincontrolled oscillations following the equilibrium. The mean and standard deviation of $G_{S}^{\prime}$ from those experiments is 11.1 and $4.2 \mathrm{~N} / \mathrm{m}$, respectively. Nevertheless, the enhanced elasticity of the stiff state cannot be only attributed to time effects because values of $\bar{G}_{S 0}^{\prime}$ are still consistently greater than $G_{S}^{\prime}$ measured in the soft state: the mean and standard deviation of $G_{S}^{\prime}$ in the soft state is 1.5 and $0.8 \mathrm{~N} / \mathrm{m}$, respectively.

To validate the accuracy of the elasticity measurements obtained from the FFT of the strain vibrations, we compare them to measurements of $G_{S}^{\prime}$ obtained from controlled oscillations. The apparent elasticity at the end of the equilibrium period $\bar{G}_{S}^{\prime}$ is calculated from FFTs performed on strain data collected during the final $20 \mathrm{~s}$ of the equilibration period (or the final $5 \mathrm{~s}$ when the equilibration period was $20 \mathrm{~s}$ ). Values of $\bar{G}_{S}^{\prime}$ show excellent agreement with linear values of $G_{S}^{\prime}$ obtained from the forced oscillations performed after the equilibration [Fig. 14(b)]. The accuracy of the $\bar{G}_{S}^{\prime}$ measurements obtained from FFTs of the strain vibrations also extends to the soft state. Immediately following the stress-oscillations of descending stress amplitude (experiments nos. 7-10), there was a period of 2-4 seconds in which the rheometer applied no stress. We performed a FFT on the $\gamma$ vibrations collected during this time period and calculated values of $\bar{G}_{S}^{\prime}$ that were in excellent agreement with linear elasticity values $G_{S}^{\prime}$ measured during the preceding forced oscillations [see open symbols in Fig. 14(b)]. To our knowledge, this is the first report of completely passive elasticity measurements, in which the perturbations responsible for the vibration arise only from the noise associated with the instrument and the environment.

The time-dependence of $\bar{G}_{S}^{\prime}$ during the equilibrium period indicates that a sample in the stiff state does not evolve to the soft state when the applied stress is zero. This observation, however, does not apply to relaxation that occurs under constant strain conditions. At the conclusion of the stress relaxation experiment, $p$ had descended to $-120 \mathrm{~Pa}$, not far from the value of about $-150 \mathrm{~Pa}$ that is associated with soft state. Although we did not perform controlled oscillations following the stress relaxation, we were able to collect about $9 \mathrm{~s}$ of passive strain vibrations from raw data that was taken less than 2 min after the conclusion of the stress relaxation, and immediately preceding the onset of the pre-shear associated with the following oscillatory tests. A Fourier transform of these vibrations indicates that $G_{S}^{\prime}=1.7 \pm 0.1 \mathrm{~N} / \mathrm{m}$ and $p=-146 \mathrm{~Pa}$. These values are representative of the soft state and indicate that the soft state can be obtained by allowing stress relaxation to occur under constant strain conditions.

\section{DISCUSSION}

Our results suggest the possibility of rationalizing the mechanism responsible for the difference between the stiff and soft states in terms of our simple visualization of Fig. 7 that treats the membrane as a series of elastic strings. When the plates are rotated and the strings are stretched to their maximum extension (about $\gamma=0.02$ ), they exert a positive pressure on the bulk liquid. If the plates are maintained at this angular displacement, the strings can only relax their tension by increasing their equilibrium length. In physical terms, this may correspond to additional oxidation that replaces strained oxide elements 
with unstrained elements. Such oxidation would likely increase the surface area of the oxide film. When the torque that was stretching the film is subsequently set to zero, the film will be less taut because it has a larger surface area. The more "baggy" or wrinkled film will exert less pressure on the bulk liquid. The wrinkled film might also exhibit a lower apparent elastic modulus because it can accommodate deformation simply by changing its shape rather than straining the oxide skin. Because of this, we expect that apparent elasticity measured in the soft state does not represent the intrinsic properties of the material but is dependent on the particular shape that the material has achieved due to the combination of both stress and oxidation in its strain history. This observation may account for the fact that measurements of $G_{S}^{\prime}$ in the soft state were different for the 20 and $40 \mathrm{~mm}$ parallel plates.

By contrast, measurements of $G_{S}^{\prime}$ in the stiff state were the same for both the 20 and $40 \mathrm{~mm}$ parallel plates. The stiff measurements are preceded by a unidirectional shear that likely makes the oxide film more taut. Measurements in a taut state are more likely to reflect the true elastic properties of the film. Measured values of $G_{S}^{\prime}$ are highest when a steady stress maintains the film in a state of maximum "tautness" during the straincontrolled oscillatory measurements performed with the HRnano.

When the steady stress $\sigma_{S E}$ is set to zero following the pre-shear, the material relaxes to a state that is less taut, and therefore exhibits values of $G_{S}^{\prime}$ and $p$ that are lower than those measured at the state of maximum tautness. Nevertheless, $G_{S}^{\prime}$ remains higher than $G_{S}^{\prime}$ measured in the soft state, and some of the stress or tautness persists throughout the equilibration time. Apparently, the internal stresses of the oxide film are "trapped" in a "metastable" or soft "glassy" state that is characterized by long relaxation times. States of metastable stress are a general feature of many soft materials and are described by the soft glassy rheology model [Sollich (1998)].

The metastable stresses of the intermediate glassy state vanish as the rheometer performs oscillations of descending $\gamma_{0}$. Based on our observations, we provide a preliminary rationalization for this effect: during strain oscillations of large amplitude $\gamma_{0}$, the "glassy stresses" or elevated $G_{S}^{\prime}$ are induced in alternating directions. As $\gamma_{0}$ decreases, it is reasonable to expect that the magnitude of the stresses that are induced in alternating directions will gradually be reduced as well. As $\gamma_{0}$ continues to decrease, the system will eventually transverse a threshold at $\gamma_{Y} \approx 0.02$, where $\gamma_{0}$ is in the linear regime and, therefore, insufficient to induce the glassy stresses in either direction. The magnitude of the glassy stresses is therefore determined by a value of $\gamma_{0}$ that is just greater than $\gamma_{Y}$. In this state, the glassy stresses are small, $G_{S}^{\prime}$ exhibits a minimum, and the material is in the soft state.

In addition to these effects, our measurements are also sensitive to time. In the absence of stress, values of $G_{S}^{\prime}$ tend to increase over time. The gradual increase in $G_{S}^{\prime}$ may be caused by additional oxidation that reinforces the mechanical strength of the oxide skin. The increase in $G_{S}^{\prime}$ with the logarithm of time indicates that $G_{S}^{\prime}$ increases quickly at the beginning of the equilibrium time, and then increases progressively more slowly as time advances. The rate of increase in $G_{S}^{\prime}$ may decrease with time as oxidation sites on the material surface become less available. The increase in $G_{S}^{\prime}$, however, need not continue indefinitely. Shear rejuvenation can "reset" the elasticity to a lower value, thereby allowing some degree of reproducibility in our measurements. The time-dependent increase in $G_{S}^{\prime}$, as well as the shear rejuvenation effect, are characteristics of thixotropy, which is commonly observed in soft materials. 


\section{CONCLUSIONS}

Our experiments demonstrate that oxides of $\mathrm{Ga}$ on liquid metal substrates in atmospheric conditions exhibit a complex variety of mechanical behavior. The importance of the oxide skin relative to the bulk of the material was demonstrated by performing oscillatory experiments using parallel plates of two different sizes. These experiments demonstrate that the stresses associated with both elasticity and yielding originate from the oxide skin, and not from the bulk of the material. We characterize the linear elasticity of the oxide skin with an apparent surface shear modulus $G_{S}^{\prime}$. Our measurements of $G_{S}^{\prime}$ are complicated by the fact that values of $G_{S}^{\prime}$ can vary by more than an order of magnitude depending on the strain history: unidirectional shear gives rise to a "stiff state" of elevated values of $G_{S}^{\prime}$, and oscillations of descending strain amplitude $\gamma_{0}$ give rise to a "soft state" of lower values of $G_{S}^{\prime}$. Additional experiments showed that $G_{S}^{\prime}$ is an increasing function of the surface stresses stored in the oxide film. The stresses in the oxide film can be monitored not only using torque measurements but also using normal force measurements, which are converted to fluid pressure $p$ values. During stress relaxation experiments, both $p$ and $\sigma_{S}$ decrease over time scales of hours. Following significant relaxation, the material exhibits the soft state. These observations show that the combination of stress and time can decrease the apparent elasticity of the oxide skin, thereby complicating rheological measurements.

A second effect that can complicate measurements is the tendency of the apparent elasticity to increase in time when no stress is applied. Although there is relaxation of internal stresses during this time period, values of $G_{S}^{\prime}$ gradually increase at a rate that is proportional to the natural logarithm of time.

The dependence of our measurements on both time and strain history have the potential to significantly complicate elasticity measurements. However, we have shown that, at least for the parallel plate geometry, the state of the material can be reset through a shear rejuvenation step that returns $G_{S}^{\prime}$ to an average value that is fairly independent of time. The mechanism by which shear can bring about these effects is not clear and will hopefully be elucidated by future measurements.

We have rationalized both the time-dependant increase and decrease in $G_{S}^{\prime}$ in terms of additional oxidation of the oxide film. Oxidation may decrease the apparent elasticity by increasing the surface area of the oxide film thereby increasing its "bagginess." However, oxidation might also reinforce the oxide film, thereby increasing the apparent elasticity. If oxidation truly is responsible for these effects, then we expect that these effects may also be present in other geometries that are typically used to characterize surface rheology.

Although our experiments were performed only on liquid metals, similar behavior may extend to a wider range of complex fluids consisting of liquids coated by solid-like shells. These results show that even in a simple geometry, under simple shear, solidcoated liquids can display complex mechanical behavior. A better understanding of the mechanisms governing the properties of solid-coated liquids will facilitate their rheological characterization, as well as the use of these materials in advanced technologies.

\section{ACKNOWLEDGMENTS}

The authors are grateful to Ryan Chiechi, Frans Spaepen, John Hutchinson, Randy Ewoldt, Gareth McKinley, Ron Larson, and Charles Zukoski for valuable discussions and feedback. This work was supported by NSF (Grant No. DMR-0602684) and Harvard MRSEC (Grant No. DMR-0213805). 


\section{References}

Chiechi, R. C., E. A. Weiss, M. D. Dickey, and G. M. Whitesides, "Eutectic gallium-indium (EGaIn): A moldable liquid metal for electrical characterization of self-assembled monolayers," Angew. Chem., Int. Ed. 47(1), 142-144 (2008).

Dickey, M. D., R. C. Chiechi, R. J. Larsen, E. A. Weiss, D. A. Weitz, and G. M. Whitesides, "Eutectic gallium-indium (EGaIn): A liquid metal alloy for the formation of stable structures in microchannels at room temperature," Adv. Funct. Mater. 18, 1-8 (2007).

Eustathopoulos, N., and B. Drevet, "Determination of the nature of metal-oxide interfacial interactions from sessile drop data," Mater. Sci. Eng., A 249, 176-183 (1998).

Ewoldt, R. H., and G. H. McKinley, "Creep ringing in rheometry or how to deal with oft-discarded data in step stress tests!," Rheol. Bull. 76, 4-6 (2007).

Hutton, J. F., "Effect of changes of surface tension and contact angle on normal force measurement with the Weissenberg rheogoniometer," Rheol. Acta 11, 70-72 (1972).

Koster, J. N., "Directional solidification and melting of eutectic GaIn," Cryst. Res. Technol. 34(9), 1129-1140 (1999).

Ricci, E., E. Arato, A. Passerone, and P. Costa, "Oxygen tensioactivity on liquid-metal drops," Adv. Colloid Interface Sci. 117, 15-32 (2005).

Sollich, P., "Rheological constitutive equation for a model of soft glassy materials," Phys. Rev. E 58, 738-759 (1998).

Struik, L. C. E., "Free damped vibrations of linear viscoelastic materials," Rheol. Acta 6(2), 119-129 (1967).

Subramaniam, A. B., M. Abkarian, L. Mahadevan, and H. A. Stone, "Non-spherical bubbles," Nature (London) 438(7070), 930 (2005).

Timoshenko, S., Theory of Elastic Stability (McGraw-Hill, New York, 1936).

Walters, K., Rheometry (Wiley, New York, 1975).

Winterstetter, T. A., and H. Schmidt, "Stability of circular cylindrical steel shells under combined loading," Thin-Walled Struct. 40(10), 893-909 (2002).

Xu, H., S. Melle, K. Golemanov, and G. Fuller, "Shape and buckling transitions in solid-stabilized drops," Langmuir 21(22), 10016-10020 (2005).

Zrnic, D., and D. S. Swatik, "On the resistivity and surface tension of the eutectic alloy of gallium and indium," J. Less-Common Met. 18, 67-68 (1969). 